EPJ Web of Conferences 16, 02008 (2011)

DOI: $10.1051 /$ epjconf/20111602008

(C) Owned by the authors, published by EDP Sciences, 2011

\title{
Development of gas absorption cells for infrared echelle spectrographs
}

\author{
L. Valdivielso ${ }^{1, a}$, P. Esparza², E.L. Martín ${ }^{3,4}$, R.E. Peale ${ }^{4}$ and D. Maukonen ${ }^{4}$ \\ ${ }^{1}$ Instituto de Astrofísica de Canarias, C/Vía Láctea, s/n, 38205 La Laguna, Tenerife, Spain \\ ${ }^{2}$ Dpto. de Química Inorgánica de la Universidad de la Laguna, Cl Francisco Sánchez, s/n, \\ 38204 La Laguna, Tenerife, Spain \\ ${ }^{3}$ CSIC-INTA Centro de Astrobiología, Ctra. Ajalvir km 4, 28850 Torrejón de Ardoz, Madrid, \\ Spain \\ ${ }^{4}$ University of Centarl Florida, Physics Department, Orlando, FI 32816, USA
}

\begin{abstract}
High-resolution spectroscopy in the near-infrared could become the leading method for discovering extra-solar planets around very low-mass stars and brown dwarfs. To help to achieve an accuracy of about $1 \mathrm{~m} / \mathrm{s}$, we are developing a gas cell which consists of a mixture of gases whose absorption spectral lines span all over the near-infrared region. We here present a summary of the working technique and the main results obtained so far.
\end{abstract}

\section{INTRODUCTION}

In the last few years, a new generation of near-infrared spectrographs with high spectral resolution and radial velocity accuracy for exoplanet detection is under development [1-3]. To obtain the required precision, iodine gas cells are used for simultaneous calibration of echelle spectra in the optical regime. However, since there are no pure gases showing a wide spectral domain in the near-infrared, an appropriate mixture of gases must be found. We are therefore searching for an optimal gas mixture, stable with time and temperature, in the near-infrared wavelength range (Y, J, H and K bands). Here we describe our laboratory experiments done to date for different gas mixtures.

\section{LABORATORY MEASUREMENTS}

In the last four years we have done 48 laboratory measurements over 28 different potential gas mixtures in the 0.8-2.5 microns wavelength range. The gases are mixed in a controlled high vacuum chamber $\left(\mathrm{N}_{2}\right)$, with manual manometric control using a mercury column. After reaching vacuum inside the cell, different partial pressures of each gas are injected and the mixture is completed with Helium gas up to atmospheric pressure. We have used different partial pressures of several gases. Also, the most promising mixtures have been measured along time in order to study its stability. For laboratory measurements we have used the Cary 5 placed on the Instituto de Astrofísica de Canarias optical laboratory. Most of the measurements have been done with a data interval of $2 \mathrm{~nm}$, which is enough for sampling the mixtures spectra.

ae-mail: lval@iac.es

This is an Open Access article distributed under the terms of the Creative Commons Attribution-Noncommercial License 3.0, which permits unrestricted use, distribution, and reproduction in any noncommercial medium, provided the original work is properly cited. 

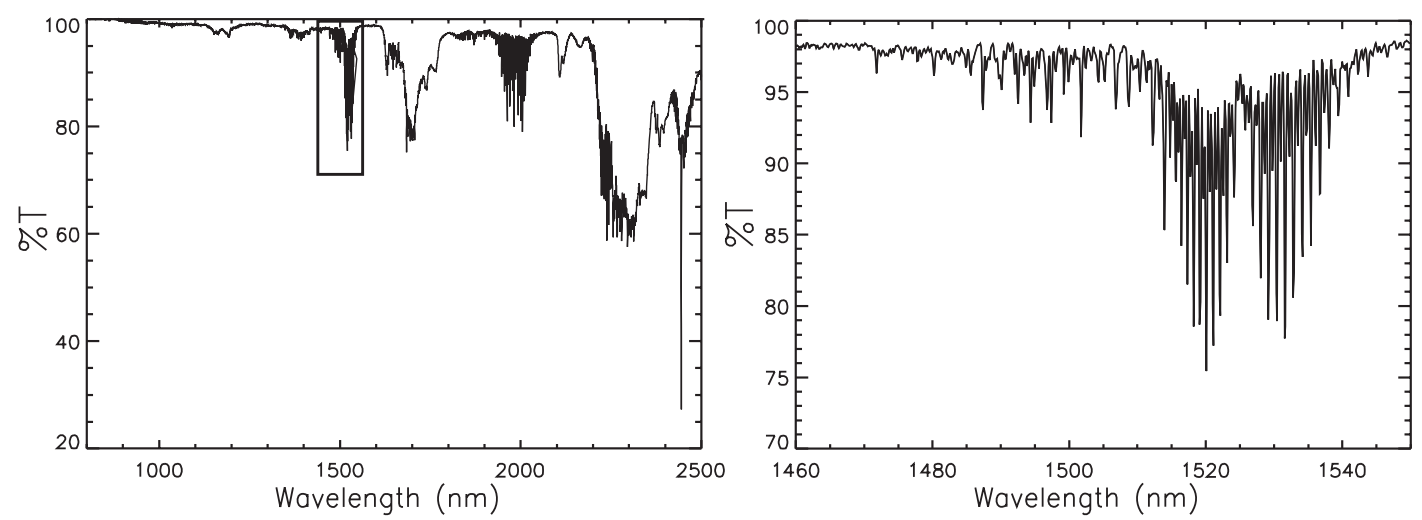

Figure 1. Left: Promising gas mixture with $\mathrm{R} \sim 30000$. Right: Zoom around $1500 \mathrm{~nm}$ corresponding to the black square in the left panel.

\section{RESULTS}

For an accurate calibration we search for a gas mixture with a forest of lines well distributed in the spectral interval between 0.9 and 2.5 microns. We have investigated 28 mixtures with acetylene, nitrous oxide, ammonia, hydrocarbons and chloromethans. In Figure 1 we show a high resolution (R 30000) promising gas mixture, although it still has few lines in the $0.9-1.5$ microns wavelength range. Mixtures with hydrocarbons, chloromethans and ammonia are pendant to be patented since they can be of interest for several projects (NAHUAL, GIANO, PRVS, SPIROU, CARMENES) and for other applications outside Astronomy, such as the characterization of new pharmaceutical products.

\section{FUTURE WORK}

We keep on working on new mixtures to try to cover the lack of lines that we find so far in the $0.95-1.5$ microns wavelength range. We have recently started with high spectral resolution measurements of the best mixtures and we have started to compile a full list of available lines. We will also keep on going with the stability study with time and temperature of the most promising gas mixtures.

This work has been supported by the Spanish Ministerio de Eduación y Ciencia through grant AYA200408271-C01.

\section{References}

[1] E. L. Martín, E. Guenther, D. Barrado y Navascués, P. Esparza, A. Manescau, U. Laux, Astronomische Nachrichten, 326, 1015 (2005)

[2] E. Oliva, L. Origlia, R. Maiolino, S. Gennari,, Ground-based Instrumentation for Astronomy (SPIE, 2004), 5492, 1274

[3] L. W. Ramsey, J. Barnes, S. L. Redman, H. R. A. Jones, A. Wolszczan, PASP, 120, 887 (2008) 